\title{
Evolution of microseismicity during frictional sliding
}

\author{
Peter Sammonds 1 and Mitiyasu Ohnaka \\ Earthquake Research Institute, University of Tokyo, Japan
}

\begin{abstract}
We have done frictional sliding experiments on Inada granite in double shear and monitored the acoustic emission (AE) produced and temporal changes in the microseismic $b$-value (where $b$ is defined as the log-linear slope of the AE frequency-amplitude distribution), using both rough and smooth ground simulated fault surfaces. We have found, (i) the maximum amplitudes of $\mathrm{AE}$ events during stable sliding are strongly dependent on the surface roughness with smooth-ground surfaces giving smaller maximum AE amplitudes; (ii) $b$-values are related to the surface topographic fractal dimensions, so that in steady-state stable sliding smooth surfaces exhibit lower $b$-values than rough surfaces; (iii) the $b$ value falls before stick-slip instability. The change of $b$ with slip we interpret in terms of evolving fractal crack damage during frictional sliding of the fault surfaces.
\end{abstract}

\section{Introduction}

A shallow earthquake is a dynamically propagating slip failure in the earth's crust. It may occur on a pre-existing fault, along a healed fault or in intact rock mass. In micromechanical terms, frictional resistance to slip for brittle rocks, where little fault gouge is present, is accompanied and accomplished by fracture of asperities on the fault surface. However, the most commonly employed theories of rock friction are phenomenological, describing friction in terms of rate and state dependent laws that take no account of the micromechanics of asperity fracture. This results from a lack of a comprehensive micromechanical model, although progress is being made through the fracture mechanics approach of Ohnaka and Kuwahara (1990), and the modelling of initial friction with contact theory by Boitnott et al. (1992) and Yoshioka and Iwasa (1996).

Fracturing of asperities produces acoustic emissions at the laboratory scale and earthquakes on a crustal scale. In recent years, the mechanics of brittle deformation have been inferred from $\mathrm{AE}$ statistics because the number of $\mathrm{AE}$ events is proportional to the number of growing cracks and $\mathrm{AE}$ amplitudes to the length of crack growth increments (e.g., Cox and Meredith, 1993). Real physical significance has been attached to these statistics and to temporal changes in the seismic $b$-value defined by the GutenbergRichter relation for the inverse earthquake cumulative frequency $N(M>m)$,

$$
\log _{10}[N(M>m)]=a-b m
$$

where $M$ is the earthquake magnitude (Richter, 1958). $\mathrm{AE}$ amplitudes measured in $\mathrm{dB}$ are divided by 20 to produce the same

\footnotetext{
'Now at Research School of Geological \& Geophysical Sciences, University College London, Gower Street, London WC1E 6BT England. (email: p.sammonds@ucl.ac.uk)
}

Copyright 1998 by the American Geophysical Union.

Paper number $98 \mathrm{GL} 00226$.

0094-8534/98/98GL-00226\$05.00 form of the relation. Results from triaxial deformation experiments on intact rock show that the $\mathrm{AE}$ rate increases exponentially up to specimen failure and that the $b$-value decreases from an initial value of 1.5 to a critical value of 0.5 at failure. These changes are associated with the formation and propagation of the shear fault which causes failure (Sammonds et al., 1992). Laboratory studies of frictional sliding undertaken in triaxial cells (Scholz, 1968; Weeks et al., 1978) have shown that for stick-slip behaviour, there is an exponential increase in $\mathrm{AE}$ event rate before the frictional instability occurs, and the $b$-value falls to a minimum of between 0.5 and 0.3 . On a crustal scale, $b$-values above 1 are measured for background seismicity, and about 0.5 for earthquake foreshocks (Smith, 1981). Recently, Main et al. (1990) have described temporal changes observed in $\mathrm{AE}$ event rate and $b$-value during cataclastic rock deformation, in terms of the evolution of fractal crack damage. They argue that the evolving distributions of crack lengths are scale invariant, that is to say they are fractal and characterized by the crack length fractal dimension $D_{L}$, with $D_{L}=2 b$ for their experiments. In seismological studies of faults, the fractal interpretation has also been validated, as the log-linear frequencymagnitude distribution is a direct consequence of the relative constancy of earthquake stress drops and a power-law distribution of fault lengths (Aki, 1981).

The purpose of the work presented here is (i), to validate, in double shear, that the $b$-value falls before stick-slip instability, (ii) that the $b$-value during steady-state stable sliding is related to fault surface roughness, and (iii) to interpret cracking during frictional sliding in terms of an evolving fractal distribution of damage. The double shear test has an advantage over the triaxial compression test on intact rock, that because deformation is already localized the shear instability at failure can be better controlled by the test machine and give higher temporal resolution of damage evolution.

\section{Experimental procedure}

We have done double shear friction experiments, where two simulated faults slide simultaneously (sandwich type), on compact blocks of Inada granite $\left(100 \times 50 \times 50 \mathrm{~mm}^{3}\right)$ with carefully prepared surfaces. The experiment is done at constant shear displacement rate $\left(6.1 \times 10^{-3} \mathrm{~mm} / \mathrm{s}\right)$ under servo-control, and the change in shear stress with displacement is monitored. Normal stress is maintained constant $(9.8 \mathrm{MPa})$ under servo-control and the fault contact area remains constant. Local strains are measured by strain gauge rosettes mounted next to the sliding surfaces and slip displacements of the surfaces by yielding-type strain gauges; the signals are amplified and filtered in the frequency range $\mathrm{DC}$ to $10 \mathrm{kHz}$, and sampled at $5 \mathrm{~Hz}$ using a 12-bit analogue to digital converter.

$\mathrm{AE}$ is detected continuously using two piezoelectric transducers mounted on the outer rock blocks, then amplified, sorted by a signal analyzer and recorded, using an instrument set-up described by Cox and Meredith (1993). The transducers have a fundamental frequency of $700 \mathrm{kHz}$, covering the principal frequency ranges of the acoustic emissions (Read et al. 1995) and we set the detecting ability of the system such that a $35 \mathrm{~dB}$ event corresponds to approximately $1 \mathrm{~mm}$ crack growth. Arrival times of $\mathrm{AE}$ events 


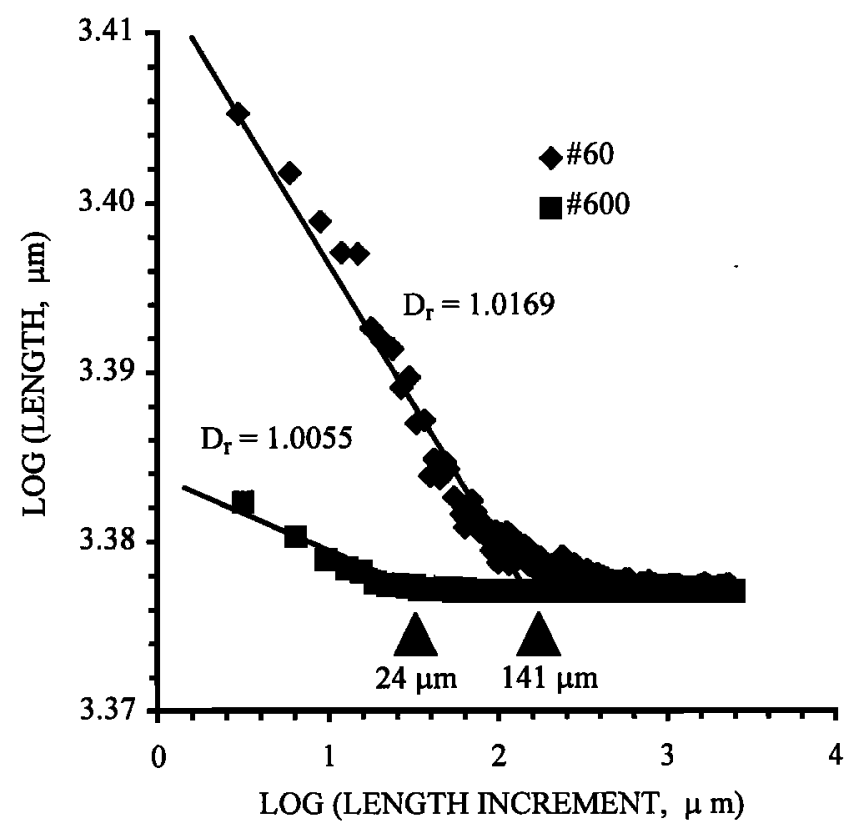

Figure 1. Plots of the $\log (L)$ against $\log (d L)$ where $L$ is the length of the surface profile and $d L$ is the incremental length measure for rough $(\# 60)$ and smooth (\#600) surfaces taken prior to sliding. For the rough surface, the topographic surface roughness fractal dimension, $D_{r}$, is 1.0169 and 1.0055 for the smooth surface. The fractal limits are $141 \mu \mathrm{m}$ and $24 \mu \mathrm{m}$ for the rough and smooth surfaces respectively.

were continuously recorded along with their (i) peak amplitudes, (ii) approximate energy, (iii) number of signal threshold crossings (usually termed counts) and their (iv) duration. The transducer arrangement remained fixed relative to the contacting surfaces throughout the experiments and there is no change in signal attenuation during experiments.
Inada granite is even textured, with a mean grain size of $0.75 \mathrm{~mm}$ and a total porosity of approximately $1 \%$. We prepared specimens from precut blocks that we ground to be planar and parallel, to within $0.02 \mathrm{~mm} / 100 \mathrm{~mm}$, using a reciprocating surface grinder. We then lapped the surfaces with Carborundum grit. Two grit sizes were used: coarse grit (\#60) with a mean particle size of $250 \mu \mathrm{m}$, and fine grit (\#600) with a mean particle size of $30 \mu \mathrm{m}$. We measured surface profiles using a diamond stylus profilometer with a resolution of $1 \mu \mathrm{m}$. Series of profiles for different profile lengths and several locations on the surfaces were taken prior to the experiment, both parallel and perpendicular to the sliding direction. In this way we checked the homogeneity of the surface preparation. Further profiles were taken after sliding. For a fractal surface, below the fractal limit the topographic surface roughness fractal dimension $D_{r}$ can be obtained from:

$$
\log L_{n}=C-\left(D_{r}-1\right) \log \left(d L_{n}\right)
$$

where $L_{n}$ is the length of the surface profile, $d L_{n}$ is the incremental length measure at order $n$, and $C$ is a constant (Mandelbrot, 1983). In Fig. $1 \log \left(L_{n}\right)$ is plotted against $\log \left(d L_{n}\right)$, for examples of both rough (\#60) and smooth (\#600) surfaces profiles taken prior to sliding. $D_{r}$ is 1.0169 for the rough surface and 1.0055 for the smooth surface and with fractal limits of $141 \mu \mathrm{m}$ and $24 \mu \mathrm{m}$ respectively, which roughly correspond to the grit sizes used for lapping and measure the roughness of the precut fault surfaces (Brown and Scholz, 1985; Lee et al., 1990). With increasing surface roughness, the fractal limit and fractal dimension increases. The fractal dimension varies somewhat depending on the value chosen for the fractal limit, but Fig. 1 clearly shows that there is a measurable difference for rough and smooth surfaces.

\section{Results}

Fig. 2 shows an example of the results from double shear experiments with rough ground (\#60) surfaces. Slip has been taken to large displacements (greater than $10 \mathrm{~mm}$ for a $100 \mathrm{~mm}$ specimen).

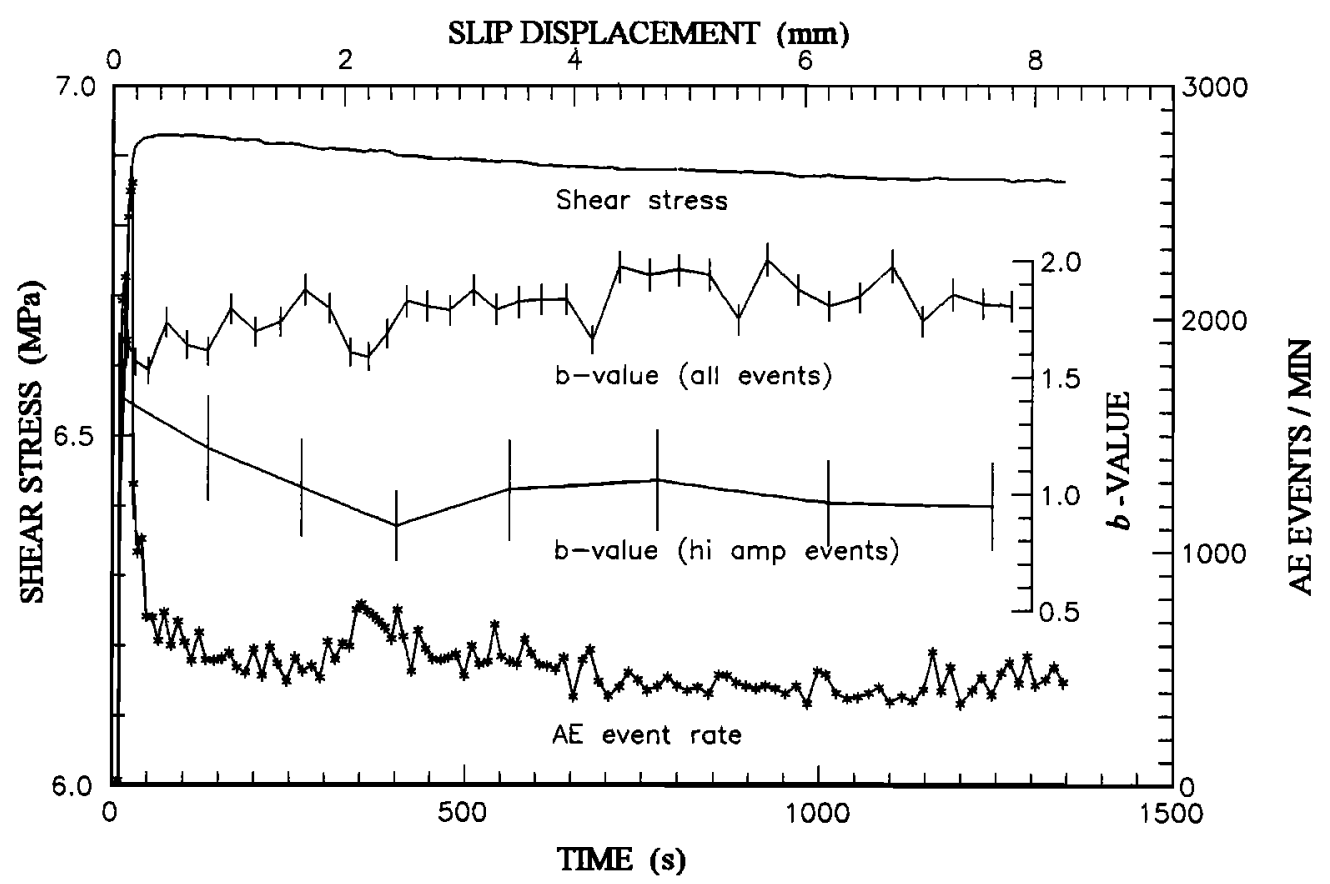

Figure 2. Results from a double shear frictional sliding experiment for blocks with rough (\#60) surfaces. Shear stress, AE event rate and $b$ value are plotted against time. The $b$-values for "all events" have been calculated for events greater than $35 \mathrm{~dB}$ and for "hi amp" events for events greater than $58 \mathrm{~dB}$. Error bars show the $95 \%$ confidence limits $(+/-1.95$ standard deviations) for the $b$-values. 


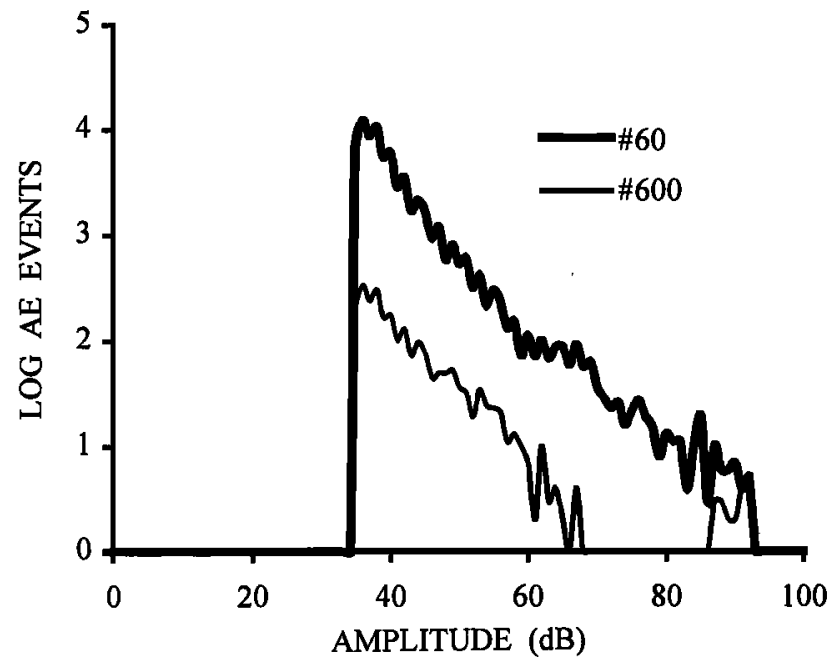

Figure. 3. The $A E$ event frequency for the experiments shown in Fig. 2 (rough \#60 surface) and Fig. 4 (smooth \#600 surface) plotted against amplitude.

Some $43000 \mathrm{AE}$ events were recorded in total during the experiment. The increase in shear stress is accompanied by a sharp increase in $\mathrm{AE}$ event rate up to a maximum, after which the $\mathrm{AE}$ rate drops rapidly to a roughly steady value of 500 events per minute. The $b$-value remains roughly steady throughout the experiment. A plot of the AE frequency-amplitude distribution (Fig. 3) shows $b$ to be two-valued: there is a larger value of $b$ for lower amplitude events and a smaller value for higher amplitude events. A plot of the temporal evolution of $b$ for events greater than $58 \mathrm{~dB}$ shows an initial decline in $b$, although the overall distribution is dominated by the greater number of small events which remains constant.

Fig. 4 shows an example of the results for smooth ground (\#600) surfaces under the same conditions of slip-rate and normal stress as the rough surface experiment. Now just some $6000 \mathrm{AE}$ events were recorded in total in the experiment. Fig. 4 shows the progress of the test up to the point where regular stick-slip is about to begin. The highest amplitude $\mathrm{AE}$ events ( 80 to $90 \mathrm{~dB}$ ) shown on the frequency-amplitude distribution, Fig. 3, are from this last portion. (Regular stick-slip itself has been excluded from the plots to exclude $\mathrm{AE}$ associated with stick-slip from the statistical calculations, as they show very different distributional characteristics). After the initial peak in the $\mathrm{AE}$ event rate, the $\mathrm{AE}$ rate above the threshold is considerably lower than for the rough surfaces. The comparison of AE amplitudes shown in Fig. 3 for the two experiments shows that for the period of steady-state stable sliding, the maximum amplitude of events is over $65 \mathrm{~dB}$ (corresponding to an inferred crack size of $50 \mu \mathrm{m}$ ) for the smooth surfaces, compared with over $85 \mathrm{~dB}$ for the rough surfaces experiment (corresponding to a crack size of $500 \mu \mathrm{m}$ ).

From Fig. 2 and 4, a comparison of the temporal evolution of $b$ can be made for the rough and smooth surfaces. The $b$-values have all been calculated for a dynamic range in excess of $20 \mathrm{~dB}$, giving confidence that $b$ is not subject to detection bias (Page, 1968). Errors bars show $+/-1.95$ standard deviations, representing the $95 \%$ confidence limits. A comparison of the $b$-values (over all events) for the initial period during steady-state stable sliding shows that the smooth surfaces yield lower $b$-values than the rough surfaces. For the smooth surfaces $b$ falls before stick-slip instability.

\section{Discussion}

In fracture mechanics terms, rupture nucleates on a fault plane at a local point of weakness, which is a consequence purely of the heterogeneous nature of rock (Ohnaka and Kuwahara, 1990). The sharp initial rise in $\mathrm{AE}$ event rate to a maximum, with increasing shear stress occurs over a short but finite time as asperities on the fault surfaces fracture. The values of $b$ we have measured and the fall in $b$ we observed prior to stick-slip are in agreement with other experimental observations (Weeks et al., 1978; Sammonds et al. 1992) and those found in nature (Smith, 1981). As the fault slides, the resultant log-linear $\mathrm{AE}$ frequency-amplitude distribution implies that there is a fractal length distribution of cracks growing as contacting asperities interact. The fact that the $b$-value for steady-state stable sliding $b$-value is lower for the smooth (\#600) surface than the rough (\#60) surface implies that the relative

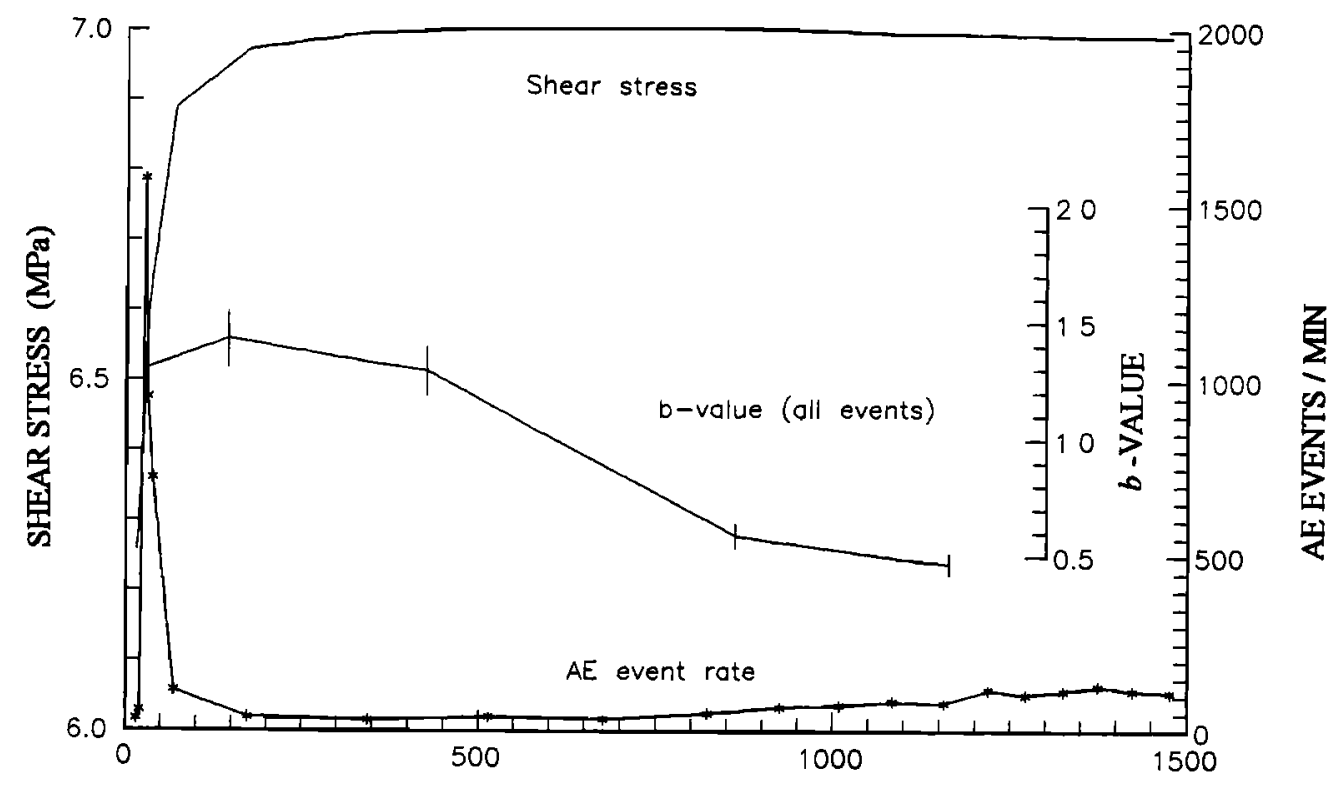

TTME (s)

Figure. 4. Results from a double shear frictional sliding experiment for blocks with smooth surfaces (\#600). Shear stress, AE event rate and $b$-value are plotted against time for events greater than $35 \mathrm{~dB}$. Error bars show the $95 \%$ confidence limits ( $+/-1.95$ standard deviations) for the $b$-values. 
frequency of large cracks to small cracks is enhanced for the smooth surface, and the enhancement progresses further as stickslip instability is approached. (The absolute size of cracks, as inferred from the $\mathrm{AE}$ amplitudes is of course larger for the rough surface).

This behaviour can be understood in terms of the surface roughness of the sliding surfaces. We have observed that the fractal limit and the topographic fractal dimension, $D_{r}$, are lower for the smooth surface than the rough surface, and the absolute size of asperity is smaller for the smooth surface. The fact that rougher surfaces have higher topographic fractal dimensions has also been reported by Lee et al. (1990) in a detailed study of rock surface profiles. Dieterich and Kilgore (1996) have measured the distribution and size of contact areas between roughened surfaces of transparent material under the microscope. They find that the density distribution of contact areas is also fractal, characterized by a contact area fractal dimension, $D_{c a}$, but that $D_{c a}$ decreases with increasing surface roughness of the contacting surfaces. They argue this implies a greater number of large contacts relative to small contacts for rough surfaces. (They measure $D_{c a}$ to be 2.64 for $\# 240$ quartz surfaces and 1.55 for $\# 60$ quartz surfaces under $10 \mathrm{MPa}$ normal stress).

On the face of it the findings of Dieterich and Kilgore might appear to conflict with our $\mathrm{AE}$ measurements: that the $b$-value is lower for smoother surfaces and decreases as stick-slip instability is approached. However as Henderson and Main (1992) have argued when modelling the evolution of seismicity along a fault it is also important to include the lengths between cracks. Although Main and Henderson treat the case of an increasing remote stress, we believe their argument is applicable to the case where the cracking is driven by the displacement of sliding surfaces under constant shear stress as in our experiments: as two fractal surfaces slide, fresh populations of asperities will be brought into contact with each resulting in increasing (and then decreasing) local stresses as the contacting asperities interact.

For contacting rough surfaces, as there are a greater number of large contacts, the length between contacts is larger than for smooth surfaces, that is to say the contacts are more isolated. This is demonstrated in the synthetic contact images of Dieterich and Kilgore. If a crack grows on an isolated part of the surface under the action of stress $\sigma$, it will be inhibited from growing further, when the crack semi-length, $a$, approaches the radius of its domain, $d$, by a local relaxation of the stress intensity, $K$ described by (Costin, 1989):

$$
K \propto \sigma[(d-a) / a] \cdot(\pi a) 1 / 2
$$

The stability of this cracking is due to negative feedback between the increase in crack length and the stress intensity. (Note the stress intensity will also decrease for constant stress). However adjacent cracks have the affect of increasing the stress intensity at the crack tips and in the region in-between. This is described by:

$$
K \propto \sigma[c \tan (\pi a / 2 c)]^{1 / 2}
$$

where $c$ is the centre-to-centre distance between adjacent cracks (Rudnicki and Kanamori, 1981). This corresponds to positive feedback between the increase in crack length and the stress intensity. Henderson and Main (1992) classify fracture processes as "persistent", "random" or "anti-persistent". The degree of persistence is indicated by the Hurst number, which is calculated as $H=n-D$, where $n$ is the Euclidean dimension of the system (Mandelbrot, 1983). For low values of $D_{c a}$ where larger more isolated but well-ordered contacts occur high values of $b$ would be expected because of the predominance of distributed stable cracks. The Hurst number for the contact area distribution should therefore be high as this is persistent behaviour, and this is found to be the case by Dieterich and Kilgore (1996). Conversely, when the stress intensity at the tip of a crack is increased by the presence of a neighbour so that the fracture continues to grow and positive feedbacks occurs, as would be the case for high values of $D_{c a}$, this would result in lower values of $b$. As frictional sliding proceeds the positive feedback and incipient instability result in a decline in $b$ value. In our experiments, for the smooth surfaces, we observe that $b$ falls significantly during slip, leading up to eventually stick-slip instability (Fig. 4).

Acknowledgments. We thank Dr M Nakatani, Mr K Motchitsuki and Dr C Stuart for their assistance. The manuscript has been much improved by the detailed criticism of Drs N. Yoshioka and I. G. Main and an anonymous reviewer. PS was a JSPS Postdoctoral Research Fellow. The research was supported by the Japan Ministry of Education, Science and Culture, the Earthquake Research Institute, the Royal Society of London and the British Council.

\section{References}

Aki, K., A probabilistic synthesis of precursory phenomena, In: Simpson, D. W. and Richards, P. G. (eds) Earthquake Prediction: an International Review. American Geophysical Union, Maurice Ewing series, 4, 566-574, 1981.

Boitnott, G.N., R.L. Biegel, C.H. Scholz, N. Yoshioka and W. Wang, Micromechanics of rock friction 2: quantitative modeling of initial friction with contact theory, J. Geophys. Res., 97, 8965-8978, 1992.

Brown, S.R. and C.H. Scholz, Closure of random elastic surfaces in contact, J. Geophys. Res., 90, 5531-5545, 1985.

Costin, L.S., Deformation and failure, in Fracture Mechanics of Rock, edited by B.K. Atkinson, pp. 167-215, Academic Press, London, 1989.

Cox, S.D. and P.G. Meredith, Microcrack formation and material softening in rock measured by monitoring acoustic emissions, Int. J. Rock Mech. Min. Sci. \& Geomech. Abstr., 30, 11-24, 1993.

Dieterich, J.H. and B.D. Kilgore, Imaging surface contacts: power law contact distributions and contact stresses in quartz, calcite, glass and acrylic plastic, Tectonophys., 256, 219-239, 1996.

Henderson, J. and Main I. A simple fracture mechanical model for the evolution of seismicity, Geophys. Res. Letts., 19, 365-368, 1992.

Lee, Y.H., J.R. Carr, D.J. Barr and C.J. Haas, The fractal dimension as a measure of the roughness of rock discontinuity profiles, Int. J. Rock. Mech. Min. Sci. \& Geomech. Abstr., 17, 453-464, 1990.

Main, I. G., P. G. Meredith, P. R. Sammonds and C. Jones, Influence of fractal flaw distribution on rock deformation in the brittle field, In: $R$. J. Knipe and E. H. Rutter (eds), Deformation Mechanics, Rheology and Tectonics, Geol. Soc. Spec. Pub. No. 54, pp. 81-96, 1990.

Mandelbrot, B.B., The Fractal Geometry of Nature, 468pp., W.H. Freeman, New York, 1983

Ohnaka, M. and Y. Kuwahara, Characteristic features of local breakdown near a crack-tip in the transition zone, Tectonophys., 175, 197-220, 1990.

Page R., Aftershocks and microaftershocks of the great Alaska earthquake of 1964, Bull. Seis. Soc. Am. 58, 1131-1168, 1968.

Read, M.D., M.R. Ayling, P.G. Meredith and S.A.F. Murrell, Microcracking during triaxial deformation of porous rocks monitored by changes in rock physical properties, Tectonophys., 245, 223-235, 1995.

Richter, C.F., Elementary Seismology. Freeman, San Francisco, 1958

Rudnicki, J.W. and H. Kanamori, Effect of fault interaction on moment stress drop and strain energy retum, $J$. Geophys. Res., 86, 1785-1793, 1981.

Sammonds, P.R., P.G. Meredith and I.G. Main, Role of pore fluids in the generation of seismic precursors to shear fracture, Nature, 359, 228230, 1992.

Scholz, C. H., Microfracturing and inelastic deformation of rock, $J$. Geophys. Res., 73, 1417-1432, 1968.

Smith, W.D., The $b$-value as an earthquake precursor, Nature, 289, 136$139,1981$.

Weeks, J., D. Lockner and J. Byerlee, Change in b-values during movement on cut surfaces in granite, Bull. Seismol. Soc. Am., 68, 333341, 1978.

Yoshioka, N. and $\mathrm{K}$. Iwasa, The characteristic displacement in rate and state-dependent friction from a micomechanical point of view, PAGEOPH, 147 (3), 433-453, 1996.

Peter Sammonds and Mitiyasu Ohnaka, Earthquake Research Institute, University of Tokyo, Bunkyo-ku, Tokyo 113 Japan.

(Received July 19, 1994; Revised September 10, 1997;

Accepted January 6, 1998) 